International Journal of

Environmental Research and

Public Health

ISSN 1660-4601

www.mdpi.com/journal/ijerph

Article

\title{
Do Placebo Response Rates from Cessation Trials Inform on Strength of Addictions?
}

\author{
Robert A. Moore ${ }^{1}$ and Henri-Jean Aubin ${ }^{2, *}$
}

1 Nuffield Division of Anaesthetics, University of Oxford, The Churchill, Oxford OX1 2JD, UK;

E-Mail: Andrew.moore@pru.ox.ac.uk

2 Hôpital Universitaire Paul Brousse, Université Paris-Sud, INSERM U699, 12 Avenue PV Couturier, BP 200, Villejuif Cedex 94804, France

* Author to whom correspondence should be addressed; E-Mail: henri-jean.aubin@pbr.aphp.fr; Tel.: +33-145-59-39-51; Fax: +33-145-59-38-63.

Received: 2 December 2011; in revised form: 6 January 2012 / Accepted: 6 January 2012 /

Published: 11 January 2012

\begin{abstract}
There is an implied assumption that addictions to different substances vary in strength from weak (easier to stop) to strong (harder to stop), though explicit definitions are lacking. Our hypothesis is that the strength of addictions can be measured by cessation rates found with placebo or no treatment controls, and that a weaker addiction would have a higher cessation rate than a stronger addiction. We report an overview of systematic reviews and meta-analyses of cessation trials, using randomised or quasi-randomised trials and reporting objectively-measured abstinence. The outcome for comparison was quit rates-typically the percentage of participants abstinent according to an objective test of abstinence at six months or longer. Twenty-eight cessation reviews (139,000 participants) were found. Most data came from reviews of smoking cessation in over 127,000 participants, and other reviews each covered a few thousand participants. Few reviews used data from studies shorter than three months, and almost all determined abstinence using objective measures. Cessation rates with placebo in randomised trials using objective measures of abstinence and typically over six months duration were $8 \%$ for nicotine, $18 \%$ for alcohol, $47 \%$ for cocaine, and $44 \%$ for opioids. Evidence from placebo cessation rates indicates that nicotine is more difficult to give up than alcohol, cocaine, and opioids. Tobacco is also a severe addiction, with a number of major deleterious health effects in a large number of people.
\end{abstract}


Keywords: tobacco; alcohol; opiates; cocaine; cannabis; addiction

\section{Introduction}

A recent review considering the definition of addiction highlighted five essential elements, which mainly addressed issues of the individuals and their behaviour and interaction with addictive stimuli [1]. Addictive interactions also involve the addictive stimulus itself, and the interactions are often described as strong or severe, without clear explanation of what strong or severe means. Here we hypothesis that it may be possible to assess the strength of an addiction in terms of how hard it is to break, and the severity of an addiction in terms of adverse physical, psychological and social effects, the duration of the addiction, and the number of people affected. It should be emphasised that a dictionary definition of hypothesis is "a supposition or proposed explanation made on the basis of limited evidence as a starting point for further investigation"; that is exactly the case here, where there is no significant prior evidence, merely an assumption that this might be reasonable.

There is an implied assumption that addictions to different substances vary in strength from weak (easier to stop) to strong (harder to stop), though explicit definitions are lacking. Using the data of the National Epidemiologic Survey on Alcohol and Related Condition, Lopez-Quintero et al. have shown that the probability of a transition from first use to dependence was greater with nicotine followed by alcohol, cocaine, and cannabis [1], and that after the onset of dependence, remission appeared usually first for cocaine, followed by cannabis, alcohol, and finally nicotine [2]. Another way to explore the strength of addictions is to measure cessation rates found with placebo or no treatment controls; a weaker addiction would have a higher cessation rate than a stronger addiction.

Comparing different forms of addiction in order to categorise the strength of addiction is not easy for reasons concerning participants, setting, and details of study design:

1. Motivation to quit and the anticipated benefit are predictors of substance cessation [3], and treatment intensity affects outcome, as shown in smoking cessation meta-analysis [4]. These can be linked and differ markedly between addictions. Alcohol dependent subjects entering treatment with current social and psychological distress that improves dramatically in days or weeks of inpatient treatment are quite different from smokers with no apparent immediate benefit trying to quit alone and at home.

2. Individual circumstances differ. An example might be individuals recruited in primary care for a short intervention for smoking cessation, compared to those in hospital with a recent diagnosis of smoking-related disease [5]. Recent smoking cessation trials have shown quit rates to be better after admission for coronary heart disease [6,7].

3. Types and intensity of intervention may be an influence, divided mainly between psychosocial interventions (cognitive behavioural, support psychotherapy) and drugs of various sorts.

4. Cessation can be defined in a number of ways. Best is to have some objective test, like breath carbon monoxide or urine cotinine for nicotine, benzoylecgonine or opioid metabolites for illicit drugs, and biochemical tests for alcohol. Self-reported abstinence is likely to be less reliable, unless confirmed by a significant other. 
5. Duration of observation is likely to be an issue. Many smokers have given up for a day; few give up for a month, and only a small percentage for a year or more. Comparing studies of different duration may be problematical.

6. Issues around size. Many studies in addiction are quite small, with a few tens of participants. We know that small studies are problematic, and that the random play of chance can have a major influence on results [8] such that results with fewer than 200 actual events may mislead [9].

The best source of information will be from published systematic reviews and meta-analyses of randomised trials of interventions to improve quit rates with information after defined periods (six months, 12 months, longest), and in which placebo or no-treatment controls are a common comparator.

\section{Methods}

Systematic reviews and meta-analyses were sought of interventions to increase quit rates. Two main searching strategies were employed:

1. Searching of Cochrane Library for Cochrane reviews, using key words tobacco, nicotine, alcohol, drinking, cocaine, heroin, opioids, and by examining lists of reviews from the appropriate review groups.

2. Searching PubMed, using similar key words in title or abstract, in humans, and in English. Related article links were also used.

Only systematic reviews or meta-analyses were used. Where several examined the same subject, the most recent and largest was chosen. Analyses of predominantly short-term outcomes were excluded because these may not provide a reliable measure of longer-term abstinence. In general, articles with cessation rates after at least six months were chosen; some included a few studies with shorter duration (three months, for example), but the proportion of short duration studies was small. Those using randomised or quasi-randomised trials were preferred; in some conditions where there was limited evidence from randomised trials, some controlled trials that were not convincingly randomised had been included in the reviews, but the amount of information from this source was a small minority of the total available. These few reviews were included to provide information where otherwise none would have been available; this was mostly in interventions for cocaine abuse, where the number of subjects for any review was small. For example, non-randomised studies contributed fewer than $1 \%$ of patients in reviews of smoking cessation.

The outcome for cessation trials was quit rates-typically the percentage of participants abstinent according to an objective test of abstinence at six months or longer. Results from each review are presented as absolute quit rates with treatment and placebo, together with a relative benefit or risk, and number needed to treat (NNT). We abstracted cessation rates for individual trials included in the reviews, and calculated overall cessation rates for intervention and placebo. For completeness, and to demonstrate that studies measuring placebo responses were sensitive in that interventions caused change in response, we also calculated relative risk with $95 \%$ confidence interval (CI) using the fixed effects models [10]; statistical significance was assumed when the 95\% CI did not include one. Numbers needed to treat (NNT) compared with placebo with $95 \%$ confidence interval were calculated from pooled data [11] only with a statistically significant results. 


\section{Results}

Table 1 contains details of and references to 12 systematic reviews for tobacco cessation studies, three for alcohol, seven for cocaine, and five for opioids. Another meta-analysis of psychosocial interventions reported results for polysubstance use, cocaine, opioids, and cannabis [12]. In total, the 28 reviews reported on almost 139,000 participants.

\subsection{Nicotine}

Information was available from 12 systematic reviews [13-24], 11 of which were Cochrane reviews, covering drug interventions and interventions including behavioural counselling, group therapy, and exercise programmes, as well as nurse or doctor interventions. Data were available on over 127,000 participants with cessation rates at six months or longer, with the bulk from reviews of nicotine replacement therapy (NRT), physical intervention, self-help, nursing interventions and bupropion. Most reviews used only properly randomised trials (one used controlled trials), used objective means to assess abstinence, and used either placebo or, in the case of behavioural interventions, used an appropriate control like a minimal intervention or brief advice.

Table 2 shows the main results in terms of percentage of abstinent participants with intervention and placebo or control. There was a consistent response for placebo, of between $3 \%$ and $14 \%$, with an overall average cessation rate of $8.4 \%$ in 57,867 participants on placebo. There appeared to be a lower cessation rate of $6.7 \%$ in 30,837 participants with placebo or minimal interventions in behavioural interventions, compared with $10.3 \%$ in 27,640 participants for placebo in drug interventions. This may reflect differences in populations studied or differences in methods, like blinding differences. Most interventions were effective to some extent, with NNTs varying between 7 (95\% CI 6 to 10) for varenicline to 65 (45 to 110) for self-help.

The very large numbers of participants in nicotine replacement therapy (NRT) studies [22] allowed investigation of a number of variables that might affect the results obtained with placebo. The first was size.

Figure 1 demonstrates a consistent cessation rate with placebo below $20 \%$, and predominantly below $10 \%$ with group sizes of 300 participants and above. It also shows very variable cessation rates of a few percent to almost $50 \%$ where group size was 100 or below. Figure 2 shows the cessation rates with placebo or control in nicotine, alcohol, cocaine and opiates.

Table 3 examines other possible influences. Neither type of NRT product (gum, patch, inhaler, lozenge, or spray) nor duration of follow up beyond six months made any difference to placebo cessation rates. Participants from smoking clinics, and those with a high level of support in a group setting achieved somewhat higher rates of cessation, though below 20\%. Twelve month studies were less effective (NNT 21; 95\% CI 18 to 25) than sixth month studies (NNT 9.4; 7.9 to 12).

This very large body of data, from many different interventions conducted in a variety of settings, shows that with placebo or no treatment after six months or longer the overall quit rate for smoking cessation is low, at about $10 \%$. The information allows the conclusion that small studies can give highly variable and misleading results, especially where the group size is below 200 participants. 
Table 1. Details of included reviews.

\begin{tabular}{|c|c|c|c|c|c|c|c|}
\hline \multicolumn{8}{|c|}{ 1. Tobacco smoking cessation reviews } \\
\hline \multirow{2}{*}{ Reference } & \multirow{2}{*}{ Interventions } & \multirow{2}{*}{ Study characteristics } & \multirow{2}{*}{ Duration } & \multirow{2}{*}{ Outcome } & \multicolumn{2}{|c|}{ Abstinence with } & \multirow{2}{*}{ Comments } \\
\hline & & & & & Intervention & Placebo & \\
\hline $\begin{array}{l}\text { Cahill and Ussher } \\
2007 \text { [13] }\end{array}$ & $\begin{array}{l}\text { Rimonabant } \\
\text { (cannabinoid } \\
\text { receptor antagonists) } \\
\text { and placebo for } \\
\text { tobacco cessation }\end{array}$ & $\begin{array}{l}\text { RCTs in adult smokers } \\
\text { Lost to follow up } \\
\text { regarded as continuing } \\
\text { smokers } \\
\text { Prolonged abstinence } \\
\text { defined biochemically at } \\
\text { each study visit }\end{array}$ & $\begin{array}{l}\text { At least } \\
6 \text { months }\end{array}$ & $\begin{array}{l}\text { Smoking status at } \\
\text { minimum of } \\
1 \text { year }\end{array}$ & $\begin{array}{l}\text { Prolonged abstinence } \\
\text { R } 20 \mathrm{mg} \text { week 50: } \\
87 / 528 \text { (16.4\%) } \\
\text { Prolonged abstinence } \\
\text { R } 5 \mathrm{mg} \text { week 50: } \\
63 / 518(11 \%)\end{array}$ & $\begin{array}{l}\text { Prolonged } \\
\text { abstinence week } \\
\text { 50: } 57 / 521(11 \%)\end{array}$ & $\begin{array}{l}\text { RR } 1.5 \\
(1.1 \text { to } 2.1) \\
\text { for } 20 \mathrm{mg}\end{array}$ \\
\hline $\begin{array}{l}\text { Gourlay et al. } \\
2004 \text { [14] }\end{array}$ & $\begin{array}{l}\text { Clonidine and } \\
\text { placebo for tobacco } \\
\text { cessation }\end{array}$ & $\begin{array}{l}\text { RCTs in adult smokers } \\
\text { Control (placebo) } \\
\text { usually involved some } \\
\text { form of behavioural } \\
\text { therapy. }\end{array}$ & $\begin{array}{l}4.5 \text { months to } \\
1 \text { year }\end{array}$ & $\begin{array}{l}\text { Smoking status } \\
\text { by a variety of } \\
\text { methods, } \\
\text { including self } \\
\text { report }\end{array}$ & $\begin{array}{l}\text { Smoking cessation at } \\
\text { longest time: } 98 / 393 \\
(25 \%)\end{array}$ & $\begin{array}{l}\text { Smoking cessation } \\
\text { at longest time: } \\
55 / 383(14.4 \%)\end{array}$ & $\begin{array}{l}\text { RR } 1.6 \\
\text { (1.2 to } 2.2 \text { ) }\end{array}$ \\
\hline \multirow[t]{2}{*}{$\begin{array}{l}\text { Hughes et al. } \\
2007 \text { [16] }\end{array}$} & $\begin{array}{l}\text { Antidepressants and } \\
\text { placebo for smoking } \\
\text { cessation }\end{array}$ & $\begin{array}{l}\text { RCTs in adult smokers } \\
\text { Control (placbeo) } \\
\text { sometimes used } \\
\text { behavioural therapy or } \\
\text { similar interventions }\end{array}$ & $\begin{array}{l}\text { At least } \\
6 \text { months } \\
\text { from start of } \\
\text { intervention }\end{array}$ & $\begin{array}{l}\text { Abstinence from } \\
\text { smoking, } \\
\text { assessed at } \\
\text { follow up by } \\
\text { various means }\end{array}$ & $\begin{array}{l}\text { Nortriptyline } \\
\text { Smoking cessation at } \\
6 \text { months or longer: } \\
100 / 480(20.8 \%)\end{array}$ & $\begin{array}{l}\text { Placebo } \\
\text { Smoking cessation } \\
\text { at } 6 \text { months or } \\
\text { longer: } 49 / 495 \\
(9.9 \%)\end{array}$ & $\begin{array}{l}\text { RR } 2.0 \\
\text { (1.5 to } 2.8 \text { ) }\end{array}$ \\
\hline & & & & & $\begin{array}{l}\text { Bupropion } \\
\text { Smoking cessation at } \\
6 \text { months or longer: } \\
1,056 / 5,557(19 \%)\end{array}$ & $\begin{array}{l}\text { Bupropion } \\
\text { Smoking cessation } \\
\text { at } 6 \text { months or } \\
\text { longer: } 417 / 4383 \\
(9.5 \%)\end{array}$ & $\begin{array}{l}\text { RR } 1.8 \\
\text { (1.6 to } 1.9 \text { ) } \\
\text { (data here for } \\
\text { clinical } \\
\text { setting, } 6 \text { and } \\
12 \text { months } \\
\text { follow up, } \\
\text { type of } \\
\text { patient) }\end{array}$ \\
\hline
\end{tabular}


Table 1. Cont.

\begin{tabular}{|c|c|c|c|c|c|c|c|}
\hline \multirow{2}{*}{ Reference } & \multirow{2}{*}{ Interventions } & \multirow{2}{*}{ Study characteristics } & \multirow{2}{*}{ Duration } & \multirow{2}{*}{ Outcome } & \multicolumn{2}{|c|}{ Abstinence with } & \multirow{2}{*}{ Comments } \\
\hline & & & & & Intervention & Placebo & \\
\hline $\begin{array}{l}\text { Cahill et al. } \\
2007 \text { [15] }\end{array}$ & $\begin{array}{l}\text { Nicotine receptor } \\
\text { partial agonists and } \\
\text { placebo for smoking } \\
\text { cessation }\end{array}$ & $\begin{array}{l}\text { RCTs in adult smokers } \\
\text { Lost to follow up } \\
\text { regarded as continuing } \\
\text { smokers } \\
\text { Control (placebo) } \\
\text { usually involved some } \\
\text { form of behavioural } \\
\text { therapy. }\end{array}$ & $\begin{array}{l}\text { Minimum } \\
\text { follow up of } \\
\text { at least } 6 \\
\text { months }\end{array}$ & $\begin{array}{l}\text { Abstinence from } \\
\text { smoking, } \\
\text { assessed at } \\
\text { follow up by } \\
\text { various means }\end{array}$ & $\begin{array}{l}\text { Varenecline } 2 \mathrm{mg} \\
\text { Smoking cessation at } \\
6 \text { months or longer: } \\
232 / 1,082(21.4 \%)\end{array}$ & $\begin{array}{l}\text { Placebo } \\
\text { Smoking cessation } \\
\text { at } 6 \text { months or } \\
\text { longer: } 75 / 941 \\
(8.0 \%)\end{array}$ & $\begin{array}{l}\text { RR } 2.7 \\
\text { (2.1 to } 3.5 \text { ) }\end{array}$ \\
\hline $\begin{array}{l}\text { Stead et al. } \\
2008 \text { [22] }\end{array}$ & $\begin{array}{l}\text { Nicotine } \\
\text { replacement therapy } \\
\text { and placebo for } \\
\text { smoking cessation }\end{array}$ & $\begin{array}{l}\text { RCTs in adult smokers } \\
\text { Lost to follow up } \\
\text { regarded as continuing } \\
\text { smokers } \\
\text { Control (placebo) } \\
\text { usually involved some } \\
\text { form of behavioural } \\
\text { therapy. }\end{array}$ & $\begin{array}{l}\text { Minimum } \\
\text { follow up of } \\
\text { at least } \\
6 \text { months }\end{array}$ & $\begin{array}{l}\text { Abstinence from } \\
\text { smoking, } \\
\text { assessed at } \\
\text { follow up by } \\
\text { various means }\end{array}$ & $\begin{array}{l}\text { All NRT/doses } \\
\text { Smoking cessation at } \\
6 \text { months or longer: } \\
3,822 / 22,711(16.8 \%)\end{array}$ & $\begin{array}{l}\text { All NRT/doses } \\
\text { Smoking cessation } \\
\text { at } 6 \text { months or } \\
\text { longer: } \\
2,115 / 20,307 \\
(10.4 \%)\end{array}$ & $\begin{array}{l}\text { RR } 1.6 \\
(1.5 \text { to } 1.7) \\
\text { Placebo } \\
\text { results } \\
\text { virtually } \\
\text { identical for } \\
\text { all modes of } \\
\text { delivery of } \\
\text { NRT }\end{array}$ \\
\hline $\begin{array}{l}\text { Lancaster and } \\
\text { Stead } 2005 \text { [17] }\end{array}$ & $\begin{array}{l}\text { Individual } \\
\text { behavioural } \\
\text { counseling }\end{array}$ & $\begin{array}{l}\text { Not all were properly } \\
\text { randomised trials (but } \\
\text { these were a minority of } \\
\text { the trials included, most } \\
\text { of which were properly } \\
\text { randomised), and with } \\
\text { versus no treatment, } \\
\text { brief advice or self-help } \\
\text { materials as the control }\end{array}$ & $\begin{array}{l}\text { Minimum } \\
\text { follow up of } \\
\text { at least } \\
6 \text { months }\end{array}$ & $\begin{array}{l}\text { Abstinence from } \\
\text { smoking, } \\
\text { assessed at } \\
\text { follow up by } \\
\text { various means }\end{array}$ & $\begin{array}{l}\text { Behavioural therapy } \\
\text { Smoking cessation at } \\
6 \text { months or longer: } \\
291 / 2,513(11.6 \%)\end{array}$ & $\begin{array}{l}\text { Control } \\
\text { Smoking cessation } \\
\text { at } 6 \text { months or } \\
\text { longer: } 195 / 2,515 \\
(7.8 \%)\end{array}$ & $\begin{array}{l}\text { RR } 1.5 \\
\text { (1.3 to } 1.8)\end{array}$ \\
\hline
\end{tabular}


Table 1. Cont.

\begin{tabular}{|c|c|c|c|c|c|c|c|}
\hline \multirow{2}{*}{ Reference } & \multirow{2}{*}{ Interventions } & \multirow{2}{*}{ Study characteristics } & \multirow{2}{*}{ Duration } & \multirow{2}{*}{ Outcome } & \multicolumn{2}{|c|}{ Abstinence with } & \multirow{2}{*}{ Comments } \\
\hline & & & & & Intervention & Placebo & \\
\hline Ussher 2005 [23] & $\begin{array}{l}\text { Supervised or } \\
\text { unsupervised } \\
\text { exercise } \\
\text { programmes }\end{array}$ & $\begin{array}{l}\text { RCTs in smokers } \\
\text { wishing to quit or recent } \\
\text { quitters }\end{array}$ & $\begin{array}{l}\text { Minimum } \\
6 \text { months }\end{array}$ & $\begin{array}{l}\text { Abstinence from } \\
\text { smoking }\end{array}$ & $\begin{array}{l}\text { Exercise } \\
\text { Smoking cessation at } \\
6 \text { months or longer: } \\
113 / 635(18 \%)\end{array}$ & $\begin{array}{l}\text { Control } \\
\text { Smoking cessation } \\
\text { at } 6 \text { months or } \\
\text { longer: } 83 / 610 \\
(14 \%)\end{array}$ & $\begin{array}{l}\text { RR } 1.2 \\
(0.9 \text { to } 1.5)\end{array}$ \\
\hline $\begin{array}{l}\text { Stead and } \\
\text { Lancaster } \\
2005[21]\end{array}$ & $\begin{array}{l}\text { Group therapy } \\
\text { versus individual } \\
\text { self help }\end{array}$ & RCTs & $\begin{array}{l}\text { Minimum } \\
6 \text { months }\end{array}$ & $\begin{array}{l}\text { Abstinence from } \\
\text { smoking by } \\
\text { measurement }\end{array}$ & $\begin{array}{l}\text { Group } \\
\text { Smoking cessation at } \\
6 \text { months or longer: } \\
249 / 2,388(10 \%)\end{array}$ & $\begin{array}{l}\text { Control } \\
\text { Smoking cessation } \\
\text { at } 6 \text { months or } \\
\text { longer: } 116 / 2,007 \\
(5.8)\end{array}$ & $\begin{array}{l}\text { RR } 1.9 \\
(1.5 \text { to } 2.3)\end{array}$ \\
\hline $\begin{array}{l}\text { Rice and Stead } \\
2008 \text { [19] }\end{array}$ & Nursing intervention & RCTs & $\begin{array}{l}\text { Minimum } \\
6 \text { months }\end{array}$ & $\begin{array}{l}\text { Abstinence from } \\
\text { smoking by } \\
\text { measurement }\end{array}$ & $\begin{array}{l}\text { Nursing } \\
\text { Smoking cessation at } \\
6 \text { months or longer: } \\
1,154 / 8,383(14 \%)\end{array}$ & $\begin{array}{l}\text { Control } \\
\text { Smoking cessation } \\
\text { at } 6 \text { months or } \\
\text { longer: } 761 / 6,822 \\
(11 \%)\end{array}$ & \\
\hline $\begin{array}{l}\text { Stead et al. } \\
2008[20]\end{array}$ & $\begin{array}{l}\text { Physician } \\
\text { intervention }\end{array}$ & RCTs & $\begin{array}{l}\text { Minimum } \\
6 \text { months }\end{array}$ & $\begin{array}{l}\text { Abstinence from } \\
\text { smoking by } \\
\text { measurement }\end{array}$ & $\begin{array}{l}\text { PhysicianSmoking } \\
\text { cessation at } 6 \text { months } \\
\text { or longer: } \\
1,029 / 12,584(8.2 \%)\end{array}$ & $\begin{array}{l}\text { ControlSmoking } \\
\text { cessation at } \\
6 \text { months or longer: } \\
470 / 9,676(4.9 \%)\end{array}$ & \\
\hline
\end{tabular}


Table 1. Cont.

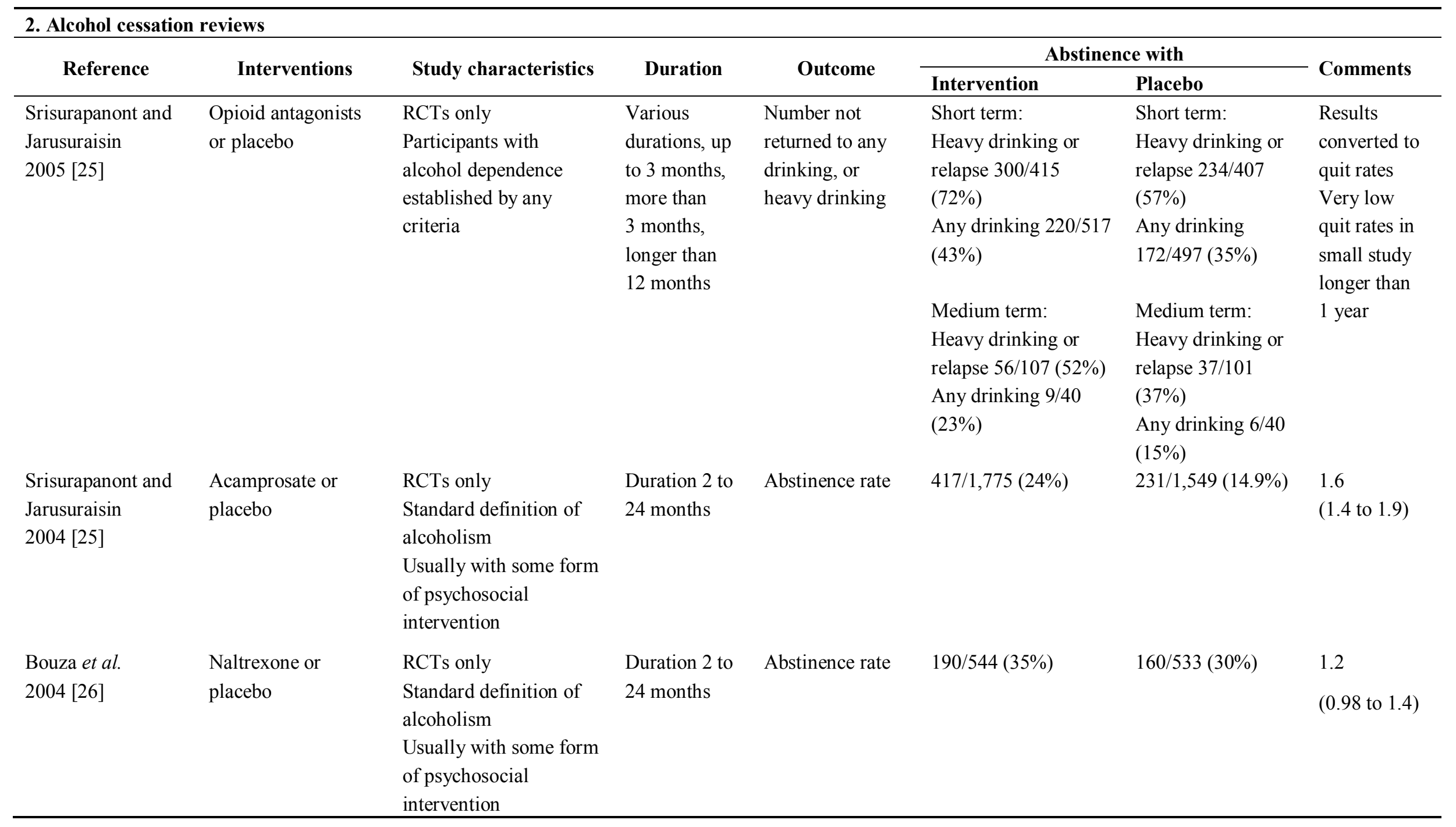


Table 1. Cont

\begin{tabular}{|c|c|c|c|c|c|c|c|}
\hline \multicolumn{8}{|c|}{ 3. Cocaine cessation reviews } \\
\hline Reference & Interventions & Study characteristics & Duration & Outcome & \multicolumn{2}{|c|}{ Abstinence with } & Comments \\
\hline $\begin{array}{l}\text { Minozzi et al. } \\
2008 \text { [27] }\end{array}$ & $\begin{array}{l}\text { Anticonvulsants and } \\
\text { placebo }\end{array}$ & $\begin{array}{l}\text { Randomised trials and } \\
\text { controlled trials } \\
\text { Cocaine dependent } \\
\text { patients (DSM } \\
\text { classification) } \\
\text { Adults }\end{array}$ & $\begin{array}{l}\text { Mean } \\
\text { duration } \\
11 \text { weeks } \\
\text { (range 1-24 } \\
\text { weeks) }\end{array}$ & $\begin{array}{l}\text { Non-use of } \\
\text { cocaine (self } \\
\text { report or } \\
\text { measurement) }\end{array}$ & $126 / 270(47 \%)$ & $102 / 198(52 \%)$ & $\begin{array}{c}\text { RR } 1.1 \\
\text { (0.9 to } 1.3 \text { ) }\end{array}$ \\
\hline $\begin{array}{l}\text { Silva de Lima et al. } \\
2003 \text { [29] }\end{array}$ & $\begin{array}{l}\text { Antidepressants and } \\
\text { placebo } \\
\text { results for } \\
\text { desipramine }\end{array}$ & $\begin{array}{l}\text { Randomised trials and } \\
\text { controlled trials } \\
\text { Cocaine dependent } \\
\text { patients (DSM } \\
\text { classification) } \\
\text { Adults }\end{array}$ & $1-6$ months & $\begin{array}{l}\text { Non-use of } \\
\text { cocaine } \\
\text { (measurement) }\end{array}$ & $59 / 136(43.3 \%)$ & $65 / 130(50 \%)$ & \\
\hline $\begin{array}{l}\text { Soares et al. } \\
2003 \text { [30] }\end{array}$ & $\begin{array}{l}\text { Dopamine agonists } \\
\text { and placebo } \\
\text { results for } \\
\text { amantadine }\end{array}$ & $\begin{array}{l}\text { Randomised trials } \\
\text { Cocaine dependent } \\
\text { patients (DSM } \\
\text { classification) } \\
\text { Adults }\end{array}$ & $\begin{array}{l}2-16 \text { weeks }+ \text {, } \\
\text { but mainly } \\
12-16 \text { weeks }\end{array}$ & $\begin{array}{l}\text { Non-use of } \\
\text { cocaine } \\
\text { (measurement) }\end{array}$ & $34 / 88(38.6 \%)$ & $34 / 127(26.8 \%)$ & \\
\hline $\begin{array}{l}\text { Amato et al. } \\
2007 \text { [31] }\end{array}$ & $\begin{array}{l}\text { Antipsychotics and } \\
\text { placebo }\end{array}$ & $\begin{array}{l}\text { Randomised trials and } \\
\text { controlled trials } \\
\text { Cocaine dependent } \\
\text { patients (DSM } \\
\text { classification) } \\
\text { Adults }\end{array}$ & 6-24 weeks & $\begin{array}{l}\text { Maintained in the } \\
\text { programme - did } \\
\text { not drop out }\end{array}$ & $62 / 106(58 \%)$ & $46 / 102(45 \%)$ & \\
\hline
\end{tabular}


Table 1. Cont.

\begin{tabular}{|c|c|c|c|c|c|c|c|}
\hline \multirow{2}{*}{ Reference } & \multirow{2}{*}{ Interventions } & \multirow{2}{*}{ Study characteristics } & \multirow{2}{*}{ Duration } & \multirow{2}{*}{ Outcome } & \multicolumn{2}{|c|}{ Abstinence with } & \multirow{2}{*}{ Comments } \\
\hline & & & & & Intervention & Placebo & \\
\hline $\begin{array}{l}\text { Knapp et al. } \\
2007 \text { [32] }\end{array}$ & $\begin{array}{l}\text { Cognitive } \\
\text { behavioural therapy } \\
\text { versus counseling }\end{array}$ & $\begin{array}{l}\text { Randomised trials } \\
\text { Cocaine dependent } \\
\text { patients (DSM } \\
\text { classification) } \\
\text { Adults }\end{array}$ & 4-6 months & $\begin{array}{l}\text { Maintained in the } \\
\text { programme-did } \\
\text { not drop out }\end{array}$ & $157 / 289(54 \%)$ & $130 / 281(46 \%)$ & \\
\hline $\begin{array}{l}\text { Castells et al. } \\
2007 \text { [33] }\end{array}$ & $\begin{array}{l}\text { Mandizol, } \\
\text { dexamphetamine, } \\
\text { methylphenidate, } \\
\text { modafinil, } \\
\text { buproprion and } \\
\text { placebo }\end{array}$ & $\begin{array}{l}\text { Randomised trials } \\
\text { Cocaine dependent } \\
\text { patients (DSM } \\
\text { classification)Adults }\end{array}$ & $1-6$ months & $\begin{array}{l}\text { Maintained in the } \\
\text { programme - did } \\
\text { not drop out }\end{array}$ & $177 / 344(51 \%)$ & $158 / 296(53 \%)$ & \\
\hline \multicolumn{8}{|c|}{ 4. Opioids cessation reviews } \\
\hline \multirow{2}{*}{ Reference } & \multirow{2}{*}{ Interventions } & \multirow{2}{*}{ Study characteristics } & \multirow{2}{*}{ Duration } & \multirow{2}{*}{ Outcome } & \multicolumn{2}{|c|}{ Abstinence with } & Comments \\
\hline & & & & & Intervention & Placebo & comits \\
\hline $\begin{array}{l}\text { Amato et al. } \\
2004 \text { [34] }\end{array}$ & $\begin{array}{l}\text { Psychosocial and } \\
\text { pharmacological } \\
\text { treatments versus } \\
\text { pharmacological } \\
\text { treatments }\end{array}$ & RCTs & $\begin{array}{l}\text { Mostly of } \\
6 \text { months or } \\
\text { more }\end{array}$ & $\begin{array}{l}\text { Number opioid } \\
\text { free at end of } \\
\text { treatment }\end{array}$ & $\begin{array}{l}\text { Psych }+ \text { Pharm } \\
\text { Opioid free at about } \\
6 \text { months: } 37 / 89 \\
(42 \%)\end{array}$ & $\begin{array}{l}\text { Pharm only } \\
\text { Opioid free at } \\
\text { about } 6 \text { months: } \\
24 / 95(25 \%)\end{array}$ & \\
\hline $\begin{array}{l}\text { Farré et al. } \\
2002[35]\end{array}$ & $\begin{array}{l}\text { Methadone, } \\
\text { buprenorphine, } \\
\text { placebo }\end{array}$ & $\begin{array}{l}\text { RCT } \\
\text { Methadone maintenance } \\
\text { at least } 12 \text { weeks } \\
\text { Various measures of } \\
\text { retention or illicit drug } \\
\text { use }\end{array}$ & $\begin{array}{l}\text { Studies } 13-40 \\
\text { weeks, mostly } \\
6 \text { months or } \\
\text { more }\end{array}$ & $\begin{array}{l}\text { Freedom from } \\
\text { illicit drug use }\end{array}$ & $\begin{array}{l}\text { Methadone: } \\
\text { 481/1,004 (52\%) } \\
\text { Buprenorphine: } \\
164 / 275(40 \%)\end{array}$ & $\begin{array}{l}\text { Placebo: } \\
65 / 131(50 \%)\end{array}$ & \\
\hline
\end{tabular}


Table 1. Cont.

\begin{tabular}{|c|c|c|c|c|c|c|c|}
\hline \multirow{2}{*}{ Reference } & \multirow{2}{*}{ Interventions } & \multirow{2}{*}{ Study characteristics } & \multirow{2}{*}{ Duration } & \multirow{2}{*}{ Outcome } & \multicolumn{2}{|c|}{ Abstinence with } & \multirow{2}{*}{ Comments } \\
\hline & & & & & Intervention & Placebo & \\
\hline $\begin{array}{l}\text { Mattick et al. } \\
2003 \text { [36] }\end{array}$ & $\begin{array}{l}\text { Methadone } \\
\text { maintenance versus } \\
\text { tapering }\end{array}$ & RCTs & $\begin{array}{l}\text { Various } \\
\text { times, largely } \\
\text { of the order of } \\
6 \text { months }\end{array}$ & $\begin{array}{l}\text { Retained in } \\
\text { treatment } \\
\text { Drug free urine }\end{array}$ & $\begin{array}{l}\text { Methadone } \\
\text { maintenance: } \\
\text { Retained 173/254 } \\
(68 \%) \\
\text { DFU: } 103 / 195(53 \%)\end{array}$ & $\begin{array}{l}\text { Tapered } \\
\text { Retained: } 63 / 251 \\
(25 \%) \\
\text { DFU: } 49 / 214 \\
(23 \%)\end{array}$ & \\
\hline $\begin{array}{l}\text { Mattick et al. } \\
2008 \text { [37] }\end{array}$ & $\begin{array}{l}\text { Buprenorphine or } \\
\text { placebo }\end{array}$ & RCTs & $\begin{array}{l}\text { Shortest } \\
4 \text { week, } \\
\text { others } \\
2 \text { months or } \\
\text { longer }\end{array}$ & $\begin{array}{l}\text { Retained in } \\
\text { treatment opioid } \\
\text { free }\end{array}$ & $\begin{array}{l}\text { Buprenorphine } \\
\text { Opioid free: } 495 / 742 \\
(67 \%)\end{array}$ & $\begin{array}{l}\text { Placebo } \\
\text { Opioid free: } \\
\text { 202/476 (42\%) }\end{array}$ & \\
\hline $\begin{array}{l}\text { Gowing et al. } \\
2006 \text { [38] }\end{array}$ & $\begin{array}{l}\text { buprenorphine, } \\
\text { clonidine, other } \\
\text { active, but not } \\
\text { placebo }\end{array}$ & $\begin{array}{l}\text { RCTs or quasi } \\
\text { randomised trials }\end{array}$ & $\begin{array}{l}\text { Mostly short } \\
\text { term }\end{array}$ & $\begin{array}{l}\text { Number } \\
\text { completing } \\
\text { programme, } \\
\text { presumably drug } \\
\text { free, but that is } \\
\text { not explicitly } \\
\text { stated }\end{array}$ & $\begin{array}{l}\text { Buprenorphine } \\
317 / 506(63 \%) \\
\text { Clonidine } \\
155 / 378(41 \%)\end{array}$ & & \\
\hline
\end{tabular}


Table 2. Results of smoking cessation reviews, ordered by number of participants. The order is by numbers of participants in the reviews. Intervention is as described in each review, and details of the reviews is in Table 1.

\begin{tabular}{|c|c|c|c|c|c|}
\hline \multirow{2}{*}{$\begin{array}{c}\text { Intervention } \\
\text { (trial six months or } \\
\text { longer) }\end{array}$} & \multirow{2}{*}{$\begin{array}{c}\text { Numbers of } \\
\text { patients }\end{array}$} & \multicolumn{2}{|c|}{ Percent abstinent with } & \multirow{2}{*}{$\begin{array}{c}\text { Relative } \\
\text { benefit } \\
(95 \% \text { CI }) \\
\end{array}$} & \multirow{2}{*}{$\begin{array}{c}\text { NNT } \\
(95 \% \text { CI })\end{array}$} \\
\hline & & Active & Placebo & & \\
\hline Nicotine & & & & & \\
\hline $\begin{array}{l}\text { replacements } \\
\text { therapy }\end{array}$ & 43,108 & 17 & 10 & $1.6(1.5$ to 1.7$)$ & $16(14$ to 17$)$ \\
\hline $\begin{array}{l}\text { Physician } \\
\text { intervention }\end{array}$ & 22,260 & 8 & 5 & $1.8(1.6$ to 2.0$)$ & $30(25$ to 37$)$ \\
\hline Self help & 19,504 & 7 & 5 & $1.3(1.2$ to 1.5$)$ & $65(45$ to 110$)$ \\
\hline Nursing intervention & 15,205 & 14 & 11 & $1.4(1.3$ to 1.5$)$ & $38(27$ to 64$)$ \\
\hline Bupropion & 9,940 & 19 & 10 & $2.0(1.8$ to 2.2$)$ & $11(9$ to 12$)$ \\
\hline Counselling & 5,028 & 12 & 8 & $1.5(1.3$ to 1.8$)$ & $26(18$ to 46$)$ \\
\hline $\begin{array}{c}\text { Group therapy } \\
\text { (versus self help) }\end{array}$ & 4,395 & 10 & 6 & $1.9(1.5$ to 2.3$)$ & $22(16$ to 33$)$ \\
\hline Varenecline & 2,023 & 21 & 8 & $2.7(2.1$ to 3.4$)$ & $7(6$ to 10$)$ \\
\hline $\begin{array}{c}\text { Cut down to quit } \\
\text { with NRT }\end{array}$ & 1,833 & 7 & 3 & $2.0(1.3$ to 3.0$)$ & $30(19$ to 74$)$ \\
\hline Exercise & 1,245 & 18 & 14 & $1.2(0.9$ to 1.5$)$ & $24(12$ to 640$)$ \\
\hline Rimonabant & 1,049 & 17 & 11 & $1.5(1.1$ to 2.1$)$ & $18(10$ to 72$)$ \\
\hline Notriptyline & 975 & 21 & 10 & $2.1(1.5$ to 2.9$)$ & $9(6$ to 16$)$ \\
\hline Clonidine & 776 & 25 & 14 & $1.7(1.3$ to 2.4$)$ & $9(6$ to 20$)$ \\
\hline
\end{tabular}

Figure 1. Cessation (quit) rates with placebo in NRT studies according to number in placebo group (size of symbol proportional to number in placebo group, inset scale) (data from Stead et al., 2008 [22]).

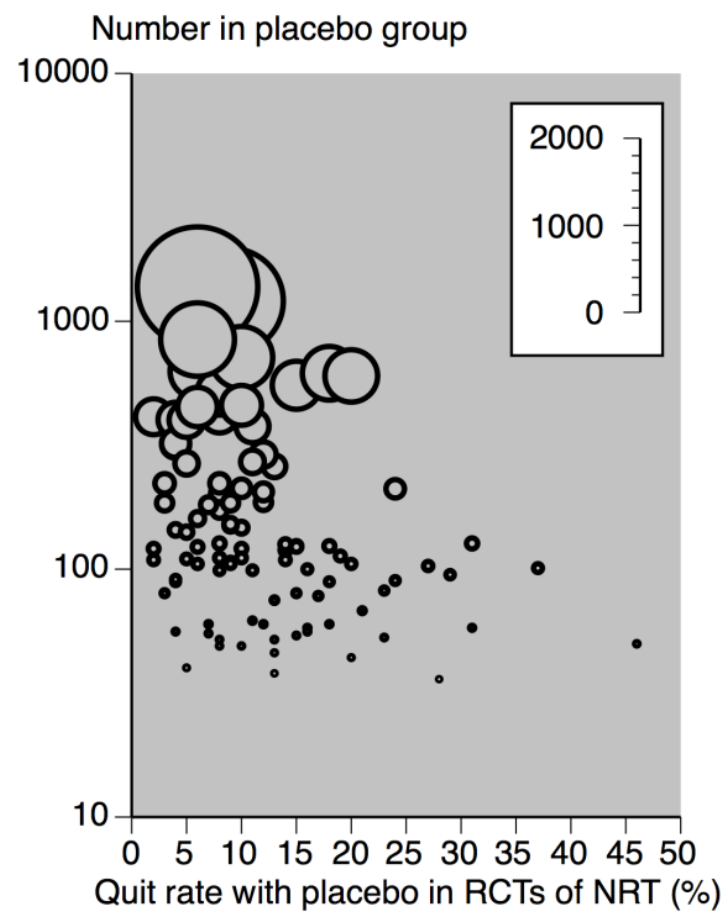


Figure 2. Cessation rates with placebo across different addictions (number of participants).

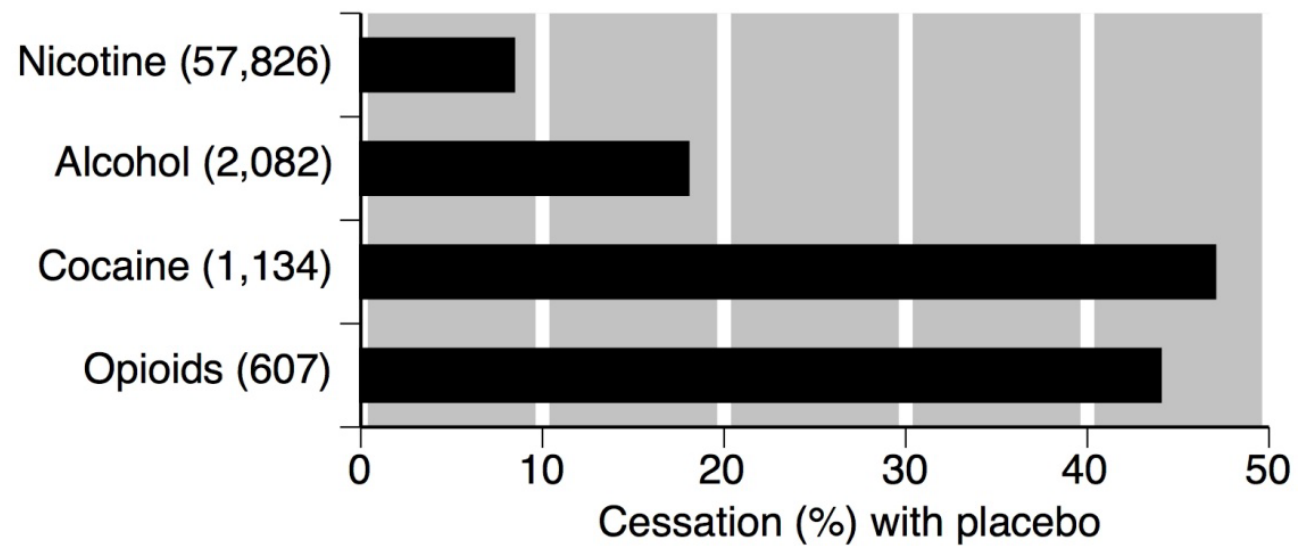

Table 3. Influence of variables in NRT trials on cessation rates with placebo. Intervention is as described in each review, and details of the reviews is in Table 1.

\begin{tabular}{|c|c|c|c|c|c|}
\hline \multirow{2}{*}{ Variable } & \multirow{2}{*}{$\begin{array}{l}\text { Numbers } \\
\text { of patients }\end{array}$} & \multicolumn{2}{|c|}{ Percent abstinent with } & \multirow{2}{*}{$\begin{array}{l}\text { Relative benefit } \\
\qquad(95 \% \text { CI })\end{array}$} & \multirow{2}{*}{$\begin{array}{c}\text { NNT } \\
(95 \% \text { CI) }\end{array}$} \\
\hline & & NRT & Placebo & & \\
\hline All trials six months or longer & 43,108 & 17 & 10 & $1.6(1.5$ to 1.7$)$ & $16(14$ to 17$)$ \\
\hline \multicolumn{6}{|l|}{ Duration of follow up } \\
\hline Six months & 4,480 & 20 & 9 & $1.9(1.6$ to 2.2$)$ & $9.4(7.9$ to 12$)$ \\
\hline Twelve months & 24,520 & 15 & 10 & $1.5(1.4$ to 1.6$)$ & $21(18$ to 25$)$ \\
\hline \multicolumn{6}{|l|}{ Trial setting } \\
\hline Community volunteers & 18,823 & 20 & 14 & $1.5(1.4$ to 1.7$)$ & $17(14$ to 20$)$ \\
\hline Smoking clinic & 1,283 & 30 & 19 & $1.6(1.3$ to 1.9$)$ & $9(7$ to 17$)$ \\
\hline Primary care & 11,427 & 11 & 7 & $1.5(1.3$ to 1.7$)$ & 25 (20 to 34$)$ \\
\hline Hospital recruitment & 3,236 & 14 & 10 & $1.3(1.04$ to 1.6$)$ & 25 (16 to 62$)$ \\
\hline \multicolumn{6}{|l|}{ Level and type of support } \\
\hline Low level of support & 12,348 & 13 & 8 & $1.6(1.4$ to 1.7$)$ & $20(16$ to 25$)$ \\
\hline High level support for individual & 16,907 & 15 & 10 & $1.5(1.4$ to 1.6$)$ & $21(17$ to 26$)$ \\
\hline High level support for group & 7,140 & 27 & 18 & $1.6(1.4$ to 1.7$)$ & $11(9$ to 14$)$ \\
\hline \multicolumn{6}{|l|}{ Type of NRT } \\
\hline Gum & 19,120 & 18 & 11 & $1.4(1.3$ to 1.5$)$ & 15 (13 to 17$)$ \\
\hline Patch & 18,175 & 16 & 10 & $1.7(1.5$ to 1.8$)$ & 17 (15 to 20$)$ \\
\hline Inhaler & 986 & 17 & 9 & $1.9(1.3$ to 2.6$)$ & $13(8$ to 28$)$ \\
\hline Lozenge or tablet & 3,109 & 16 & 8 & $2.0(1.6$ to 2.5$)$ & $12(10$ to 17$)$ \\
\hline Nasal spray & 887 & 24 & 12 & $2.0(1.5$ to 2.7$)$ & $8(6$ to 14$)$ \\
\hline
\end{tabular}

\subsection{Alcohol}

Information was available from two systematic reviews $[25,26]$ with data on over 4,600 participants (predominantly acamprosate and naltrexone [26]) were used, with cessation rates between three and 12 or 24 months; one review of opioid antagonists had 208 participants in longer-term studies [25]. Both used only properly randomised trials, used various means to assess abstinence, and used placebo, usually with some form of psychosocial intervention. 
Table 4 shows the main results in terms percentage of abstinent participants with intervention and placebo. The quit rate with placebo in the two larger reviews was $15 \%$ and $30 \%$, with an overall average cessation rate of $18 \%$ in 2,082 participants on placebo. Only acamprosate had sufficient information to demonstrate effectiveness, with an NNT of 12 (95\% CI 9 to 17).

Table 4. Results of alcohol cessation reviews, ordered by number of participants. The order is by numbers of participants in the reviews. Intervention is as described in each review, and details of the reviews is in Table 1.

\begin{tabular}{|c|c|c|c|c|c|}
\hline \multirow{2}{*}{ Intervention } & \multirow{2}{*}{$\begin{array}{c}\text { Number of } \\
\text { patients }\end{array}$} & \multicolumn{2}{|c|}{ Percent abstinent with } & \multirow{2}{*}{$\begin{array}{c}\text { Relative benefit } \\
(95 \% \mathrm{CI})\end{array}$} & \multirow{2}{*}{$\begin{array}{c}\text { NNT } \\
(95 \% \text { CI }) \\
\end{array}$} \\
\hline & & Active & Placebo & & \\
\hline Acamprosate & 3,324 & 23 & 15 & $1.6(1.4$ to 1.8$)$ & $12(9$ to 17$)$ \\
\hline Naltrexone & 1,077 & 35 & 30 & $1.2(1.0$ to 1.4$)$ & not calculated \\
\hline Opioid antagonists & 208 & 52 & 37 & $1.4(1.0$ to 2.0$)$ & $6(3$ to 43$)$ \\
\hline
\end{tabular}

\subsection{Cocaine}

Information was available from seven systematic reviews [27-33], six of which were Cochrane reviews. Six reviews examined drug therapies, and one cognitive behavioural therapy.

There was data on over 2,600 participants with cessation rates ranging between one and six months, though most comparisons were relatively small in size. All used only properly randomised trials in participants with defined cocaine addiction, used a mixture of non-use of cocaine by measurement or retention in a programme to assess abstinence, and used placebo or counselling. Studies using validated non-use by measurement or maintenance in programme appeared not to differ in cessation rates with placebo.

Table 5. Results of cocaine cessation reviews, ordered by number of participants. The order is by numbers of participants in the reviews. Intervention is as described in each review, and details of the reviews is in Table 1.

\begin{tabular}{|c|c|c|c|c|c|}
\hline \multirow{2}{*}{ Intervention } & \multirow{2}{*}{$\begin{array}{c}\text { Number } \\
\text { of patients }\end{array}$} & \multicolumn{2}{|c|}{ Percent abstinent with } & \multirow{2}{*}{$\begin{array}{c}\text { Relative benefit } \\
(95 \% \text { CI) } \\
\end{array}$} & \multirow{2}{*}{$\begin{array}{c}\text { NNT } \\
(95 \% \text { CI })\end{array}$} \\
\hline & & Active & Placebo & & \\
\hline CNS stimulants & 640 & 51 & 53 & $1.0(0.8$ to 1.1$)$ & not calculated \\
\hline $\begin{array}{c}\text { Cognitive } \\
\text { behavioural therapy }\end{array}$ & 570 & 54 & 46 & $1.2(1.0$ to 1.4$)$ & not calculated \\
\hline Anticonvulsants & 468 & 47 & 52 & $0.9(0.8$ to 1.1$)$ & not calculated \\
\hline Carbamazepine & 313 & 39 & 32 & $1.3(0.9$ to 1.7$)$ & not calculated \\
\hline Desipramine & 266 & 43 & 50 & $0.9(0.7$ to 1.1$)$ & not calculated \\
\hline Amantadine & 215 & 39 & 27 & $1.4(1.0$ to 2.1$)$ & not calculated \\
\hline Antipsychotics & 208 & 58 & 45 & $1.3(1.0$ to 1.7$)$ & not calculated \\
\hline
\end{tabular}

Table 5 shows the main results in terms percentage of abstinent participants with intervention and placebo, and the relative benefit. The quit rate with placebo varied between $27 \%$ and $53 \%$, with an overall average cessation rate of $47 \%$ in 1,134 participants on placebo (not double counting carbamazepine results with those of anticonvulsants). A review of psychosocial interventions had a 
placebo quit rate of $21 \%$ in a small number of small trials [12]. No intervention was significantly better than placebo.

\subsection{Opioids}

Information was available from five systematic reviews [34-38], four of which were Cochrane reviews. Three reviews included data of six months or longer, and two [37,38] of one to six months. There were a variety of interventions, including psychosocial and pharmacological, mainly methadone or buprenorphine. The reviews mainly used only properly randomised trials in participants with defined opioid addiction, used a mixture of non-use of opioid by measurement or retention in a programme to assess abstinence, and used placebo, or, in one review, maintenance methadone versus tapered withdrawal. There was information on over 2,300 participants with cessation rates over various times.

Table 6 shows the main results in terms percentage of abstinent participants with intervention and placebo. For methadone, results are taken from a review [35] reporting true placebo and not contaminated with tapering methadone dose. For psychosocial plus pharmacological interventions, the placebo response included pharmacological interventions and may not be a true placebo, and some of the buprenorphine trials used clonidine as a control. The quit rate with placebo varied between $25 \%$ and $50 \%$, with an overall average cessation rate of $43 \%$ in 590 participants on true placebo in methadone and buprenorphine trials. A review of psychosocial interventions had a placebo quit rate of $24 \%$ in a small number of small trials [12]. Only buprenorphine showed any efficacy in a sensible number of patients, with an NNT of 4.6 (95\% CI 3.6 to 6.6$)$.

Table 6. Results of opioid cessation reviews, ordered by number of participants. The order is by numbers of participants in the reviews. Intervention is as described in each review, and details of the reviews is in Table 1.

\begin{tabular}{|c|c|c|c|c|c|}
\hline \multirow{2}{*}{ Intervention } & \multirow{2}{*}{$\begin{array}{c}\text { Number of } \\
\text { patients }\end{array}$} & \multicolumn{2}{|c|}{ Percent abstinent with } & \multirow{2}{*}{$\begin{array}{c}\text { Relative benefit } \\
(95 \% \text { CI })\end{array}$} & \multirow{2}{*}{$\begin{array}{c}\text { NNT } \\
(95 \% \text { CI }) \\
\end{array}$} \\
\hline & & Active & Placebo & & \\
\hline Methadone & 1,135 & 48 & 50 & $1.0(0.8$ to 1.7$)$ & not calculated \\
\hline Buprenorphine & 884 & 63 & 41 & $1.7(1.5$ to 2.0$)$ & $4.6(3.6$ to 6.6$)$ \\
\hline $\begin{array}{l}\text { Psychosocial plus } \\
\text { pharmacological }\end{array}$ & 184 & 42 & 25 & $1.7(1.1$ to 2.6$)$ & $6.1(3.4$ to 35$)$ \\
\hline
\end{tabular}

\subsection{Cannabis}

The abstinence rate in a single review [12] of mostly short term psychotherapeutic interventions for cannabis dependence was $15 \%$ in a small number of small trials.

\section{Discussion}

Cessation rates with true placebo in randomised trials using objective measures of abstinence and typically over six months duration were $8 \%$ for nicotine, $18 \%$ for alcohol, $47 \%$ for cocaine, and $43 \%$ for opioids. This overview of systematic reviews sought evidence of different quit rates with placebo 
in addictions to different substances, and apparently found it. Before accepting such a result at face value, it is necessary to explore how robust it is.

By concentrating on data from systematic reviews and meta-analyses of randomised trials reporting abstinence at six months or longer it relied on studies least open to bias. Most information came from reviews of smoking cessation in over 127,000 participants, though reviews for treatments of other addictions covered a few thousand participants. Most of the reviews included had a preponderance of longer-term studies, with determination of abstinence using objective measures. As much as possible, therefore, comparisons were of like for like.

The example of nicotine replacement therapy, with over 43,000 participants in trials of six months or longer, showed that vagaries of trial design made little difference to placebo response rates, though trials lasting 12 months were less effective than those lasting only six months. Together, these approaches support the contention that between-addiction comparisons of quit rates with placebo are justified.

Unresolved issues include how missing data are treated in clinical trials; missing data should probably be counted as failure, but this may not be uniformly applied, and is not generally discussed. Trials with cocaine addicts had shorter durations than with other substances. As the abstinence rate tends to decrease with time, this bias is of concern.

The use of a no-treatment control rather than actual placebo might be important in both non-drug and drug interventions. Placebo has been shown to produce genuine effects through psychological mechanism (involving expectations, conditioning learning, memory, motivation somatic focus, reward, anxiety reduction, and meaning), as well as changes of metabolic activity in different brain regions in cocaine abusers [39]. Context effects could also be a source of variation in placebo response rates $[40,41]$. However, placebo response rates tend to be consistent in particular randomised trial models, using the same outcomes, over the same period of time [42], differing only when the outcome differs [43-45]. In the case of addiction, the same outcome was being sought over the same time.

We have shown a high variability of success rates in smoking cessation trials where the group size is below 200 subjects. Trials with fewer than 200 participants were over-represented in opiate and cocaine studies.

Finally, studies included in the systematic reviews were sensitive in that interventions caused change in response, with statistically significant relative benefit. This should give comfort that placebo response measured were meaningful. Few interventions were shown to be highly effective, and low NNTs were rare, except for buprenorphine treatment for opioid addiction, where one opioid replaces another (Table 5).

The conclusion, then, is that in the circumstances chosen, placebo quit rates are a useful proxy marker for the strength of different addictions. The results point to tobacco being by far the strongest, as has already suggested the findings from epidemiological data [1,2].

Tobacco addiction is far more widespread than other addictions. Many smokers are not alcohol or drug addicts, though most alcohol or drug addicts are also smokers. One can hypothesize on individual vulnerability to addictive behaviours, this vulnerability being highest in alcohol and drug addicts. One other approach could be to look at differential outcomes in tobacco and other substance cessation in subjects having a dual addiction. In this respect, it has been shown that alcohol outcome was far better than tobacco outcome in alcoholic smokers undergoing an alcohol and tobacco concurrent 
intervention [46]. Everyday practice shows that many alcoholics consider that quitting tobacco to be far more difficult to quit than alcohol. Likewise, polydrug abusers often consider that tobacco would be the last substance they would be able to quit.

It is also the case that, of all abused substances, nicotine is the one where the risk of developing a dependence syndrome is the highest after first exposure [1,47,48]. Even this leaves us problems, as the authors themselves use conflicting language [47]: "Over 80\% of those who had used tobacco six or more times met dependence criteria...It appears that tobacco readily produces dependence (perhaps more so than most other substances), yet it does not progress to severe levels of dependence as readily as cocaine, heroin and most other drugs. Though it easily causes compulsive use, tolerance, and withdrawal, tobacco may be less likely to get "out of control" and progress to severe dependence than most other drugs".

Perhaps the biggest difficult is that, with addiction, there are several dimensions. These include the swiftness with which an addiction takes hold, the severity or otherwise of deleterious effects, the time course of their development, whether they are balanced by any possible beneficial effects, how many are affected, and how easy it is for an addiction to be broken. In all of these dimensions, tobacco addiction rates high. It is a strong addiction, being of rapid onset and hard to break, as evidenced by the large amount of good quality evidence of low placebo response rates in cessation trials, and of major public health concern because of its negative effects on health in a large number of people.

\section{Acknowledgments}

This work was funded by Pfizer.

\section{Conflict of Interest}

This work had its genesis in discussions between RAM and Pfizer personnel about how addictions might be differentiated. That resulted in a piece of commissioned work relating to the use of placebo. The authors were free to publish the results of any research if they chose, free of any constraints by Pfizer, which had no role in design, planning, or execution of the study, or in writing the manuscript. RAM is funded by NIHR Biomedical Research Centre Programme, and has received research funding from Pfizer. HJA has received sponsorship to attend scientific meetings, speaker honorariums and consultancy fees from Pfizer, McNeil, GlaxoSmithKline, Pierre-Fabre Santé, Sanofi-Aventis, and Merck-Lipha.

\section{References}

1. Lopez-Quintero, C.; Cobos, J.P.; Hasin, D.S.; Okuda, M.; Wang, S.; Grant, B.F.; Blanco, C. Probability and predictors of transition from first use to dependence on nicotine, alcohol, cannabis, and cocaine: Results of the National Epidemiologic Survey on Alcohol and Related Conditions (NESARC). Drug Alcohol Depend. 2010, 115, 120-130. 
2. Lopez-Quintero, C.; Hasin, D.S.; de Los Cobos, J.P.; Pines, A.; Wang, S.; Grant, B.F.; Blanco, C. Probability and predictors of remission from life-time nicotine, alcohol, cannabis or cocaine dependence: Results from the National Epidemiologic Survey on Alcohol and Related Conditions. Addiction 2010, 106, 657-669.

3. Miller, W.R.; Rollnick, S. Motivational Interviewing: Preparing People for Change, 2nd ed.; Guilford Press: New York, NY, USA, 2002.

4. Clinical Practice Guideline Treating Tobacco Use and Dependence 2008 Update Panel, Liaisons, and Staff. A clinical practice guideline for treating tobacco use and dependence: 2008 update. A U.S. Public Health Service report. Am. J. Prev. Med. 2008, 35, 158-176.

5. Moore, R.A.; Gavaghan, D.J.; Edwards, J.E.; Wiffen, P.; McQuay, H.J. Pooling data for number needed to treat: No problems for apples. BMC Med. Res. Methodol. 2002, 2, doi:10.1186/1471-2288-2-2.

6. Dornelas, E.A.; Sampson, R.A.; Gray, J.F.; Waters, D.; Thompson, P.D. A randomized controlled trial of smoking cessation counseling after myocardial infarction. Prev. Med. 2000, 30, 261-268.

7. Quist-Paulsen, P.; Gallefoss, F. Randomised controlled trial of smoking cessation intervention after admission for coronary heart disease. Br. Med. J. 2003, 327, 1254-1257.

8. Moore, R.A.; Gavaghan, D.; Tramer, M.R.; Collins, S.L.; McQuay, H.J. Size is everythingLarge amounts of information are needed to overcome random effects in estimating direction and magnitude of treatment effects. Pain 1998, 78, 209-216.

9. Flather, M.D.; Farkouh, M.E.; Pogue, J.M.; Yusuf, S. Strengths and limitations of meta-analysis: Larger studies may be more reliable. Control Clin. Trials 1997, 18, 568-579; discussion 661-566.

10. Morris, J.A.; Gardner, M.J. Calculating confidence intervals for relative risks (odds ratios) and standardised ratios and rates. Br. Med. J. 1988, 296, 1313-1316.

11. Cook, R.J.; Sackett, D.L. The number needed to treat: A clinically useful measure of treatment effect. Br. Med. J. 1995, 310, 452-454.

12. Dutra, L.; Stathopoulou, G.; Basden, S.L.; Leyro, T.M.; Powers, M.B.; Otto, M.W. A meta-analytic review of psychosocial interventions for substance use disorders. Am. J. Psychiatry 2008, 165, 179-187.

13. Cahill, K.; Ussher, M. Cannabinoid type 1 receptor antagonists (rimonabant) for smoking cessation. Cochrane Database Syst. Rev. 2009, doi:10.1002/14651858.CD005353.pub3.

14. Gourlay, S.G.; Stead, L.F.; Benowitz, N.L. Clonidine for smoking cessation. Cochrane Database Syst. Rev. 2008, doi:10.1002/14651858.CD000058.pub2.

15. Cahill, K.; Stead, L.F.; Lancaster, T. Nicotine receptor partial agonists for smoking cessation. Cochrane Database Syst. Rev. 2011, doi:10.1002/14651858.CD006103.pub5.

16. Hughes, J.R.; Stead, L.F.; Lancaster, T. Antidepressants for smoking cessation. Cochrane Database Syst. Rev. 2010, doi:10.1002/14651858.CD000031.pub3.

17. Lancaster, T.; Stead, L.F. Individual behavioural counselling for smoking cessation. Cochrane Database Syst. Rev. 2008, doi:10.1002/14651858.CD001292.pub2.

18. Lancaster, T.; Stead, L.F. Self-help interventions for smoking cessation. Cochrane Database Syst. Rev. 2009, doi:10.1002/14651858.CD001118.pub2.

19. Rice, V.H.; Stead, L.F. Nursing interventions for smoking cessation. Cochrane Database Syst. Rev. 2009, doi:10.1002/14651858.CD001118.pub2. 
20. Stead, L.F.; Bergson, G.; Lancaster, T. Physician advice for smoking cessation. Cochrane Database Syst. Rev. 2008, doi:10.1002/14651858.CD000165.pub3.

21. Stead, L.F.; Lancaster, T. Group behaviour therapy programmes for smoking cessation. Cochrane Database Syst. Rev. 2009, doi:10.1002/14651858.CD001007.pub2.

22. Stead, L.F.; Perera, R.; Bullen, C.; Mant, D.; Lancaster, T. Nicotine replacement therapy for smoking cessation. Cochrane Database Syst. Rev. 2008, doi:10.1002/14651858.CD000146.pub3.

23. Ussher, M. Exercise interventions for smoking cessation. Cochrane Database Syst. Rev. 2008, doi:10.1002/14651858.CD002295.pub3.

24. Wang, D.; Connock, M.; Barton, P.; Fry-Smith, A.; Aveyard, P.; Moore, D. 'Cut down to quit' with nicotine replacement therapies in smoking cessation: A systematic review of effectiveness and economic analysis. Health Technol. Assess. 2008, 12, iii-iv, ix-xi, 1-135.

25. Srisurapanont, M.; Jarusuraisin, N. Opioid antagonists for alcohol dependence. Cochrane Database Syst. Rev. 2010, doi:10.1002/14651858.CD001867.pub3.

26. Bouza, C.; Angeles, M.; Munoz, A.; Amate, J.M. Efficacy and safety of naltrexone and acamprosate in the treatment of alcohol dependence: A systematic review. Addiction 2004, 99, 811-828.

27. Minozzi, S.; Amato, L.; Davoli, M.; Farrell, M.; Lima Reisser, A.A.; Pani, P.P.; Silva de Lima, M.; Soares, B.; Vecchi, S. Anticonvulsants for cocaine dependence. Cochrane Database Syst. Rev. 2009, doi:10.1002/14651858.CD006754.pub2.

28. Lima, A.R.; Lima, M.S.; Soares, B.G.; Farrell, M. Carbamazepine for cocaine dependence. Cochrane Database Syst. Rev. 2009, doi:10.1002/14651858.CD002023.pub2.

29. Silva de Lima, M.; Farrell, M.; Lima Reisser, A.A.; Soares, B. Antidepressants for cocaine dependence. Cochrane Database Syst. Rev. 2011, 2, doi:10.1002/14651858.CD002950.pub3.

30. Soares, B.G.; Lima, M.S.; Reisser, A.A.; Farrell, M. Dopamine agonists for cocaine dependence. Cochrane Database Syst. Rev. 2008, doi:10.1002/14651858.CD003352.

31. Amato, L.; Minozzi, S.; Pani, P.P.; Davoli, M. Antipsychotic medications for cocaine dependence. Cochrane Database Syst. Rev. 2010, doi:10.1002/14651858.CD006306.pub2.

32. Knapp, W.P.; Soares, B.G.; Farrel, M.; Lima, M.S. Psychosocial interventions for cocaine and psychostimulant amphetamines related disorders. Cochrane Database Syst. Rev. 2008, doi:10.1002/14651858.CD003023.pub2.

33. Castells, X.; Casas, M.; Vidal, X.; Bosch, R.; Roncero, C.; Ramos-Quiroga, J.A.; Capella, D. Efficacy of central nervous system stimulant treatment for cocaine dependence: A systematic review and meta-analysis of randomized controlled clinical trials. Addiction 2007, 102, 1871-1887.

34. Amato, L.; Minozzi, S.; Davoli, M.; Vecchi, S.; Ferri, M.; Mayet, S. Psychosocial and pharmacological treatments versus pharmacological treatments for opioid detoxification. Cochrane Database Syst. Rev. 2011, doi:10.1002/14651858.CD005031.pub4.

35. Farre, M.; Mas, A.; Torrens, M.; Moreno, V.; Cami, J. Retention rate and illicit opioid use during methadone maintenance interventions: A meta-analysis. Drug Alcohol Depend. 2002, 65, 283-290.

36. Mattick, R.P.; Breen, C.; Kimber, J.; Davoli, M. Methadone maintenance therapy versus no opioid replacement therapy for opioid dependence. Cochrane Database Syst. Rev. 2009, doi:10.1002/14651858.CD002209.pub2. 
37. Mattick, R.P.; Kimber, J.; Breen, C.; Davoli, M. Buprenorphine maintenance versus placebo or methadone maintenance for opioid dependence. Cochrane Database Syst. Rev. 2008, doi:10.1002/14651858.CD002207.pub3.

38. Gowing, L.; Ali, R.; White, J. Buprenorphine for the management of opioid withdrawal. Cochrane Database Syst. Rev. 2009, doi:10.1002/14651858.CD002025.pub4.

39. Finniss, D.G.; Kaptchuk, T.J.; Miller, F.; Benedetti, F. Biological, clinical, and ethical advances of placebo effects. Lancet 2010, 375, 686-695.

40. Di Blasi, Z.; Harkness, E.; Ernst, E.; Georgiou, A.; Kleijnen, J. Influence of context effects on health outcomes: A systematic review. Lancet 2001, 357, 757-762.

41. Hrobjartsson, A.; Gotzsche, P.C. Is the placebo powerless? Update of a systematic review with 52 new randomized trials comparing placebo with no treatment. J. Intern. Med. 2004, 256, 91-100.

42. Miller, F.G.; Kaptchuk, T.J. The power of context: Reconceptualizing the placebo effect. $J . R$. Soc. Med. 2008, 101, 222-225.

43. Barden, J.; Edwards, J.E.; McQuay, H.J.; Moore, R.A. Pain and analgesic response after third molar extraction and other postsurgical pain. Pain 2004, 107, 86-90.

44. Moore, R.A.; McQuay, H.J. Bandolier's Little Book of Understanding Medical Evidence; Oxford University Press: Oxford, UK, 2006.

45. Oldman, A.D.; Smith, L.A.; McQuay, H.J.; Moore, R.A. Pharmacological treatments for acute migraine: Quantitative systematic review. Pain 2002, 97, 247-257.

46. Kalso, E.; Moore, R.A. Five easy pieces on evidence-based medicine (2). Eur. J. Pain 2000, 4, 321-324.

47. Cooney, N.L.; Litt, M.D.; Cooney, J.L.; Pilkey, D.T.; Steinberg, H.R.; Oncken, C.A. Concurrent brief versus intensive smoking intervention during alcohol dependence treatment. Psychol. Addict. Behav. 2007, 21, 570-575.

48. Woody, G.E.; Cottler, L.B.; Cacciola, J. Severity of dependence: Data from the DSM-IV field trials. Addiction 1993, 88, 1573-1579.

(C) 2012 by the authors; licensee MDPI, Basel, Switzerland. This article is an open access article distributed under the terms and conditions of the Creative Commons Attribution license (http://creativecommons.org/licenses/by/3.0/). 